\title{
THE EUCLID-FOURIER-MUKAI ALGORITHM FOR ELLIPTIC SURFACES*
}

\author{
MARCELLO BERNARDARA ${ }^{\dagger}$ AND GEORG HEIN B $^{\ddagger}$
}

\begin{abstract}
We describe the birational correspondences, induced by the Fourier-Mukai functor, between moduli spaces of semistable sheaves on elliptic surfaces with sections, using the notion of $P$-stability in the derived category. We give explicit conditions to determine whether these correspondences are isomorphisms. This is indeed not true in general and we describe the cases where the birational maps are Mukai flops. Moreover, this construction provides examples of new compactifications of the moduli spaces of vector bundles via sheaves with torsion and via complexes. We finally get for any fixed dimension an isomorphism between the Picard groups of the moduli spaces.
\end{abstract}

Key words. Fourier-Mukai transform, moduli spaces, Mukai flops, elliptic surfaces, stable sheaves, Postnikov-stability.

AMS subject classifications. 14F05, 14J27, 14J60.

1. Introduction. Let $X \rightarrow \mathbb{P}^{1}$ be a $\mathrm{K} 3$ elliptic surface with a section and at most finitely many nodal singular fibers, and $\widehat{X} \rightarrow \mathbb{P}^{1}$ its relative generalized Jacobian. The relative Poincaré bundle gives a Fourier-Mukai equivalence $\Psi: \mathrm{D}^{\mathrm{b}}(X) \rightarrow \mathrm{D}^{\mathrm{b}}(\widehat{X})$. In this paper, we consider moduli spaces $M_{X}(v)$ of semistable sheaves with a fixed Mukai vector $v$ on $X$ and we describe how we can fix a Mukai vector $\hat{v}$ on $\widehat{X}$ in order to describe via $\Psi$ a birational map $\varepsilon$ between $M_{X}(v)$, and $M_{\widehat{X}}(\hat{v})$ and we study the properties of this map.

Moduli spaces of semistable sheaves on elliptic surfaces have been intensively studied in the last twenty years. The cornerstone in the theory is [4]. In that paper, the rank two case is studied, and most of the basic notions are introduced. For example, the use of a fiberlike (called suitable there) polarization (see also [3, 10]), and the description of a birational map between moduli spaces. In the case of an elliptic surface with a section $\sigma$, Friedman considered the moduli spaces of rank 2 sheaves with odd fiber degree. Without loss of generality, one can fix the Mukai vector $v$ corresponding to the Chern classes $(2, \sigma-t f, 1)$, where $t$ is a nonnegative integer and $f$ denotes the fiber. Then Friedman shows that $M_{X}(v)$ is smooth, projective, $2 t$ dimensional and birational to $\operatorname{Hilb}^{t}(X)$. Moreover, for $t=1,2$ the birational map is actually an isomorphism. He then conjectures ([4, Part III, Conjecture 4.13]) that it is an isomorphism for all $t$.

The point of view of Fourier-Mukai transforms has been introduced into this problem by Bridgeland [2]. Consider $X$ and the relative Jacobian $Y:=J_{X}(a, b)$, that is the elliptic surface whose fibers are the moduli spaces of rank $a$, degree $b$ semistable sheaves on the corresponding elliptic fiber of $X$. Bridgeland constructs a derived equivalence $\Psi: \mathrm{D}^{\mathrm{b}}(X) \rightarrow \mathrm{D}^{\mathrm{b}}(Y)$ as the Fourier-Mukai functor whose kernel is the relative universal sheaf. He extends the result of Friedman describing, for any Mukai vector $v$ with rank $r$ and fiber degree coprime, a birational map between $M_{X}(v)$ and $\operatorname{Hilb}^{t}(Y)$, where $2 t$ is the dimension of $M_{X}(v)$ and $a$ and $b$ in the definition of $Y$

\footnotetext{
*Received November 27, 2012; accepted for publication February 19, 2013.

${ }^{\dagger}$ Univeristät Duisburg-Essen, Fakultät für Mathematik. Universitätstr. 2, 45117 Essen, Germany. Current address: Institut de Mathématiques de Toulouse (IMT), 118 route de Narbonne, F-31062 Toulouse Cedex 9, France (mbernard@math.univ-toulouse.fr).

$\ddagger$ Univeristät Duisburg-Essen, Fakultät für Mathematik, Universitätstrasse 2, 45117 Essen, Germany (georg.hein@uni-due.de).
} 
depend on $v$. Moreover, he shows that these two spaces are isomorphic for small values of $t$. Remark that this extends the result of Friedman (since $Y \cong X$ if $X$ has a section) to arbitrary rank, but does not include the case $t=r=2$.

The new point of view introduced in this paper is to give a purely homological interpretation of the stability by considering a stability condition in the derived category $\mathrm{D}^{\mathrm{b}}(X)$, called $P$-stability, defined in $[6,7]$, which recovers the slope stability and is well behaved with respect to derived equivalences. A $P$-stability datum is roughly determined by fixing the dimensions of the morphism spaces in $\mathrm{D}^{\mathrm{b}}(X)$ between an object and a finite number of fixed objects in the category plus an orthogonality condition. This is encoded in a $P$-datum. In the case where $C$ is a smooth projective curve and $X \rightarrow C$ is a fibration whose generic fiber is a smooth projective curve, we provide in Remark 2.16 an explicit $P$-datum corresponding to the $\mu$-stability with respect to a fiberlike polarization. The most important feature of the $P$-datum is the orthogonality condition, which ensures the stability of a torsion free sheaf with a given Mukai vector and is well-behaved under relative equivalences. Moreover moduli spaces of $P$-stable objects (if they exist) are preserved under triangulated equivalences. The interest of our results is then twofold: the study of birational maps between moduli spaces and the construction of moduli spaces of objects in the derived category.

Consider then $X \rightarrow \mathbb{P}^{1}$ a K3 elliptic surface with a section and at most finitely many nodal singular fibers, and a Mukai vector $v=(r, \Lambda, c)$, with $r>0$ and such that $M_{X}(v)$ is smooth and projective of even dimension $2 t$. We denote by $-d$ the fiber-degree of $\Lambda$, we have $-d$ and $r$ coprime and we can assume without loss of generality that $0<d<r$. We focus our attention on the Fourier-Mukai equivalence $\Psi: \mathrm{D}^{\mathrm{b}}(X) \rightarrow \mathrm{D}^{\mathrm{b}}(\widehat{X})$ (in Bridgeland's notations, $\left.\widehat{X}=J_{X}(1,0)\right)$. We consider a Mukai vector $\hat{v}$ on $\widehat{X}$, uniquely determined by $v$. This equivalence induces a birational map

$$
\varepsilon: M_{X}(v) \rightarrow M_{\widehat{X}}(\hat{v}) .
$$

Remark that the existence of a section of $X \rightarrow \mathbb{P}^{1}$ gives an isomorphisms $X \cong \widehat{X}$. However, we think it clarifies the exposition if we distinguish between both objects.

The notion of orthogonality described in Proposition 2.13 is the key to prove the main results of this paper. Remark that we restrict, in order to have simple notations, to the case where $X$ is K3. This restriction should not be necessary, because it is used throughout the paper only to simplify numerical computations. The stated results should then hold for any elliptic surface, even though the numerical data have to be changed.

THEOREM 3.1. If $t<r$, the moduli spaces $M_{X}(v)$ and $M_{\widehat{X}}(\hat{v})$ are isomorphic and the isomorphism is induced by the Fourier-Mukai transform $\Psi$.

Notice that if $X$ has two sections a similar isomorphism has been constructed by Yoshioka [13, Thm. 3.15] in the case where the rank of $\hat{v}$ is zero.

TheOREM 4.1. If $r \leq t<r+d$, the Fourier-Mukai equivalence $\Psi$ induces a Mukai flop between $M_{X}(v)$ and $M_{\widehat{X}}(\hat{v})$, unless $t=r=2$, in which case it induces an isomorphism.

The existence of a birational map gives in particular that the Picard groups of the two moduli spaces are isomorphic.

Corollary 3.15. For all $t$, the birational map $\varepsilon: M_{X}(v) \rightarrow M_{\widehat{X}}(\hat{v})$ induces an isomorphism

$$
\operatorname{Pic}\left(M_{X}(v)\right) \simeq \operatorname{Pic}\left(M_{\widehat{X}}(\hat{v})\right) .
$$


The choice of working with a very particular dual elliptic fibration is not restrictive. We construct indeed an algorithm which recovers Bridgeland's result (Thm. 3.13) by ours in a finite number of steps. Such algorithm is obtained combining the Euclidean algorithm applied to the pair rank, fiber degree and the Fourier-Mukai transform and, starting with a 2 -dimensional moduli space of semistable sheaves whose rank and fiber degree are coprime, it ends with the Hilbert scheme $\operatorname{Hilb}^{t}(\widehat{X})$. We call it the Euclid-Fourier-Mukai algorithm. Remark that a version of this algorithm for a single elliptic curve is used in Polishchuk's book [11] to recover Atiyah's classification [1] of indecomposable vector bundle on an elliptic curve. In our case, by means of the Euclid-Fourier-Mukai algorithm, we can show that the bound given by Bridgeland coincides with ours and is indeed optimal for $r \geq 3$. Finally we argue how to find a counterexample to Friedman's conjecture ([4, III, Conjecture 4.13]), that is how to find two birational but non isomorphic moduli spaces in the case the rank is two. (take $t=4$ and $r=2$ ). Another important application of the Euclid-FourierMukai algorithm gives an isomorphism between $\operatorname{Pic}\left(M_{X}(v)\right)$ and $\operatorname{Pic}\left(\operatorname{Hilb}^{t}(\widehat{X})\right)$ for all integers $t$.

When $\varepsilon$ is not an isomorphism, our construction allows to describe new compactifications for the moduli spaces of vector bundles. One of the good properties of $P$-stability is indeed that, once we have a fine moduli space $M$ for $P$-stable objects and a triangulated equivalence $\Pi$, we have a fine moduli space of $\Pi(P)$-stable objects which is isomorphic to $M$.

Suppose $\varepsilon$ is not an isomorphism, and recall that we can describe $M_{X}(v)$ as a moduli space of $P$-stable objects in $\mathrm{D}^{\mathrm{b}}(X)$. The moduli space $N_{\widehat{X}}$ of $\Psi(P)$-stable objects in $\mathrm{D}^{\mathrm{b}}(\widehat{X})$ is a smooth irreducible projective variety, isomorphic to $M_{X}(v)$, containing sheaves with torsion and sharing a dense open subset with $M_{\widehat{X}}(\hat{v})$. On the other hand, consider the relative quasi-inverse functor $\Phi$ of $\Psi$ and the $P$-stability on $\mathrm{D}^{\mathrm{b}}(\widehat{X})$ corresponding to semistability of torsion free sheaves with Mukai vector $\hat{v}$. The moduli space $N_{X}$ of $\Phi(P)$-stable objects on $X$ is isomorphic to $M_{\widehat{X}}(\hat{v})$, shares with $M_{X}(v)$ a dense open subset, and contains complexes with two non-vanishing cohomologies. It follows that $N_{X}$ and $N_{\widehat{X}}$ provide new compactifications of the moduli space of semistable vector bundles via complexes and torsion sheaves respectively.

The paper is organized as follows: in Section 2, given a smooth projective surface $X \rightarrow C$ fibering over a smooth projective curve, and Chern classes $c_{t}=\left(r, c_{1}, c_{2}\right)$ with $r$ coprime to the fiber degree, we describe the $P$-stability corresponding to the semistability of sheaves with Chern classes $c_{t}$. The first main ingredient of this construction is the existence of a polarization $H$ on $X$ with respect to which the semistability of a torsion free sheaf with Chern classes $c_{t}$ coincides with the semistability of its restriction to the generic fiber. The second main ingredient is the description of semistability on a smooth projective curve via orthogonality with respect to some vector bundle. The main result is stated in Propositions 2.13 and 2.17. In Remark 2.16 we phrase these results in terms of Postnikov-stability. In section 3 and 4, we consider an elliptic K3 surface $X$ and use the $P$-stability to prove respectively Theorems 3.1, and 4.1. Moreover, in Section 3 we describe the Euclid-Fourier-Mukai algorithm, compare our result with Bridgeland's one, and show how to deduce the isomorphism between the Picard groups.

We work exclusively over the field $\mathbb{C}$ of complex numbers. 
Acknowledgment. This work has been supported by the SFB/TR 45 "Periods, moduli spaces and arithmetic of algebraic varieties". The authors thank Alina Marian and Dragos Oprea for writing [9], which gave the motivation to study the isomorphism between the Picard groups, and for their helpful and careful questions. It is a pleasure to thank David Ploog for comments on an early version of this article. The authors thank the anonymous referees for their useful comments and suggestions.

2. Postnikov stability data for fibered surfaces. Let $X$ be a smooth projective surface and $\pi: X \rightarrow C$ a fibration over a smooth projective curve. In this section, we show that, once we fix Chern classes $c_{t}=\left(r, c_{1}, c_{2}\right)$ such that the fiber degree of $c_{1}$ and $r$ are coprime, we can give a $P$-datum with respect to which an object in $\mathrm{D}^{\mathrm{b}}(X)$ is stable if and only if it is a semistable torsion free sheaf with Chern classes $c_{t}$ and hence fixed Hilbert polynomial. To do this, we observe that there is a polarization such that the restriction of a stable torsion free sheaf to the generic fiber has to be stable. This idea is due to Friedman [3] and further developed by himself [4] and O'Grady [10]. In what follows we generalize those results. We can even provide an upper bound on the number of fibers where the restriction of a given semistable sheaf can be unstable. Moreover, semistability on a curve corresponds to orthogonality to some vector bundle, as shown by Popa [12]. The first part of the $P$-stability is then given by an orthogonality condition with respect to a finite number of vector bundles each one supported on a single fiber. The second part of the $P$-datum constrains the object in the derived category to be a torsion free sheaf with fixed Hilbert polynomial.

2.1. Postnikov stability. We recall the definition of Postnikov stability from $[6,7]$. To start with, we define a convolution of objects $C_{m}, C_{m-1}, \ldots, C_{0}$ in a triangulated category to be a diagram

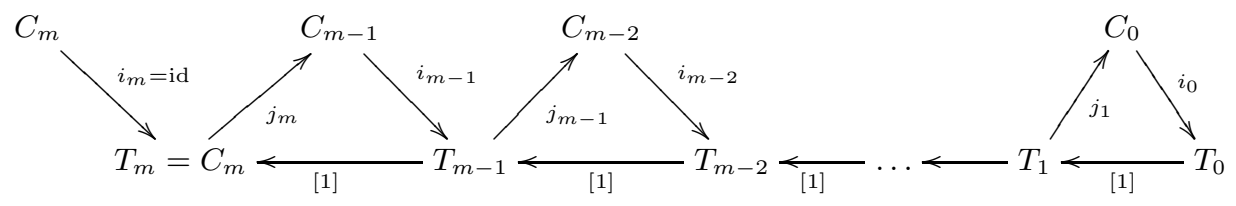

where all the triangles $T_{k+1} \rightarrow C_{k} \rightarrow T_{k} \stackrel{[1]}{\longrightarrow} T_{k+1}$ [1] are distinguished. In [5, IV.2.10] this is called a left Postnikov system.

Definition 2.1. Let $\mathcal{T}$ be a $k$-linear triangulated category, $\left\{C_{i}\right\}_{i=-n \ldots m}$ a finite collection of objects from $\mathcal{T}$, and $N_{i}^{j}$ natural numbers with $j \in \mathbb{Z}$ and $i=-n \ldots m$, with almost all $N_{i}^{j}$ equal to zero. We say that an object $E \in \mathcal{T}$ is Postnikov stable with respect to $\left(C_{i}, N_{i}^{j}\right)$ if

1. For all integers $i, j$ we have $\operatorname{dim}_{k} \operatorname{Hom}\left(C_{i}, E[j]\right)=N_{i}^{j}$, and

2. there exists a convolution of the objects $C_{m}, \ldots, C_{0}$ such that $\operatorname{Hom}\left(T_{0}, E[j]\right)=0$ for all $j \in \mathbb{Z}$.

We will shortly say $P$-datum instead of Postnikov datum.

The main motivation for considering $P$-stability is that it is preserved by equivalences and covers the classical semistability of sheaves. As an example we have following comparison theorem from [7].

THEOREM 2.2. ([r, Theorem 10]) Let $\left(X, \mathcal{O}_{X}(1)\right)$ be a smooth projective variety over $k$, and $p$ be a Hilbert polynomial. Then there exists a $P$-datum $\left(C_{i}, N_{i}^{j}\right)$ in $\mathrm{D}^{\mathrm{b}}(X)$ such that $E \in \mathrm{D}^{\mathrm{b}}(X)$ is P-stable if and only if

1. the complex $E$ is isomorphic to a sheaf $E_{0}$, 
2. $E_{0}$ is a pure sheaf of Hilbert polynomial $p$,

3. $E_{0}$ is slope semistable with respect to $\mathcal{O}_{X}(1)$.

Once we know the existence of a fine moduli space for a given $P$-datum, an equivalence of triangulated categories gives rise to another $P$-datum with isomorphic moduli space.

Proposition 2.3. Let $X$ and $Y$ be smooth projective varieties and $\Phi: \mathrm{D}^{\mathrm{b}}(X) \rightarrow$ $\mathrm{D}^{\mathrm{b}}(Y)$ an equivalence. Consider an Hilbert polynomial $p(m)$ on $X$ such that the moduli space $M_{X}(p(m))$ of semistable sheaves with Hilbert polynomial $p(m)$ on $X$ is fine. Fix on $X$ the $P$-datum corresponding to the Hilbert polynomial $p(m)$. Then there exists a fine moduli space of $\Phi(P)$-stable objects on $Y$ isomorphic to $M_{X}(p(m))$.

Proof. It is clear that $E$ in $\mathrm{D}^{\mathrm{b}}(X)$ is $P$-stable if and only if $\Phi(E)$ is $\Phi(P)$-stable. If $\mathcal{E}$ is the universal sheaf on $M_{X}(p(m)) \times X$, then $\Phi(\mathcal{E})$ is the universal object for moduli on $Y$.

2.2. Generalizing a result of O'Grady. Let $f$ denote the class of a smooth fiber of $\pi: X \rightarrow C$.

Definition 2.4. Let $E$ be a vector bundle on $X$ of rank $r$ with Chern roots $\left\{\alpha_{i}\right\}_{i=1, \ldots, r}$. The discriminant $\Delta(E)$ of $E$ is defined as follows

$$
\Delta(E):=\sum_{1 \leq i<j \leq r}\left(\alpha_{i}-\alpha_{j}\right)^{2}=(r-1) c_{1}^{2}(E)-2 r c_{2}(E) .
$$

We included the definition because many authors (as for example [8]) use other scaling constants for $\Delta(E)$. For example O'Grady includes in his definition of $\Delta$ the factor $\frac{-1}{2 r}$ (see Section IV. in [10]). With our definition we have $\Delta(E)=\operatorname{ch}_{2}(E \otimes$ $\left.E^{\vee}\right)$. The Bogomolov inequality says that when $E$ is semistable with respect to some polarization, then $\Delta(E) \leq 0$.

LEMMA 2.5. (Compare with Lemma IV.1 in [10]) Let $X$ be a smooth projective surface, $0 \rightarrow E^{\prime} \rightarrow E \rightarrow E^{\prime \prime} \rightarrow 0$ be a short exact sequence of semistable coherent sheaves of the same slope with respect to some polarization on $X$. Let $A=r^{\prime} c_{1}(E)-$ $r c_{1}\left(E^{\prime}\right)$ where $r^{\prime}$ and $r$ are the ranks of $E^{\prime}$ and $E$ respectively. Then we have an inequality

$$
\frac{r^{2}}{4} \Delta(E) \leq A^{2}
$$

Proof. The semistability of $E^{\prime}$ and $E^{\prime \prime}$ yields $c_{2}\left(E^{\prime}\right) \geq \frac{r^{\prime}-1}{2 r^{\prime}} c_{1}^{2}\left(E^{\prime}\right)$, and $c_{2}\left(E^{\prime \prime}\right) \geq$ $\frac{r-r^{\prime}-1}{2\left(r-r^{\prime}\right)} c_{1}^{2}\left(E^{\prime \prime}\right)$. The short exact sequence yields two equations

$$
c_{1}(E)=c_{1}\left(E^{\prime}\right)+c_{1}\left(E^{\prime \prime}\right), \quad \text { and } \quad c_{2}(E)=c_{1}\left(E^{\prime}\right) c_{1}\left(E^{\prime \prime}\right)+c_{2}\left(E^{\prime}\right)+c_{2}\left(E^{\prime \prime}\right) .
$$

The two inequalities give now

$$
c_{2}(E) \geq c_{1}\left(E^{\prime}\right) c_{1}\left(E^{\prime \prime}\right)+\frac{r^{\prime}-1}{2 r^{\prime}} c_{1}^{2}\left(E^{\prime}\right)+\frac{r-r^{\prime}-1}{2\left(r-r^{\prime}\right)} c_{1}^{2}\left(E^{\prime \prime}\right) .
$$

Now we express $c_{1}\left(E^{\prime \prime}\right)$ as $c_{1}(E)-c_{1}\left(E^{\prime}\right)$, and replace $c_{1}\left(E^{\prime}\right)$ by $\frac{1}{r}\left(r^{\prime} c_{1}(E)-A\right)$ to obtain

$$
2 r c_{2}(E) \geq(r-1) c_{1}^{2}(E)-\frac{1}{r^{\prime}\left(r-r^{\prime}\right)} A^{2} .
$$


Having in mind that $\Delta(E) \leq 0$, and that $r^{\prime} \in\{1,2, \ldots, r-1\}$ we obtain the stated inequality.

Lemma 2.6. Let $(X, H)$ be a smooth polarized surface, $f$ and $A$ divisors on $X$ such that $f^{2}=0, H . A=0$, and $H . f>0$. Then we have $A^{2} \leq-\frac{(A . f)^{2} H^{2}}{(H . f)^{2}}$.

Proof. We consider the Hodge index theorem for the two line bundles $H$ and $(A+\lambda f)$ and obtain $H^{2}(A+\lambda f)^{2} \leq(H . A+\lambda H . f)^{2}$. Thus, the quadratic equation $(H . f)^{2} \lambda^{2}-2 H^{2}(A . f) \lambda-H^{2} A^{2}=0$ in $\lambda$ has at most one rational solution. Considering the discriminant of this quadratic equation, we obtain the stated inequality.

Let us fix Chern classes $c_{t}=\left(r, c_{1}, c_{2}\right)$ such that $f . c_{1}$ is coprime with $r$. The following result is a generalization of O'Grady's result [10, Proposition I.1.6] and the proof follows almost literally the original one.

Proposition 2.7. Let $E$ be a torsion free coherent sheaf $E$ with Chern classes $c_{t}$ and set $h:=H . f$, and $N_{0}:=-\frac{r^{2} \Delta(E) h}{8}$. For any $N \geq N_{0}$ the following facts are equivalent:

1. E is slope stable with respect to $H+N \cdot f$.

2. $E$ is slope semistable with respect to $H+N \cdot f$.

3. $E$ is slope stable with respect to $H+N_{0} \cdot f$.

4. $E$ is slope semistable with respect to $H+N_{0} \cdot f$.

5. The restriction of $E$ to a general fiber of $\pi$ is stable.

Proof. The main ingredient of the proof is the following Lemma.

LEMMA 2.8. For any $N^{\prime} \geq N_{0}$, the sheaf $E$ cannot be strictly semistable with respect to $H+N^{\prime} \cdot f$.

Proof. Suppose $E$ is strictly semistable, that is there exists a semistable subsheaf $E^{\prime} \subset E$ of the same slope. Let $r^{\prime}$ be the rank of $E^{\prime}$, and set $A=r^{\prime} c_{1}(E)-r c_{1}\left(E^{\prime}\right)$. We have by Lemma 2.5 the inequality $\frac{r^{2} \Delta(E)}{4} \leq A^{2}$. Applying Lemma 2.6 to the polarization $H+N^{\prime} \cdot f$ we obtain $A^{2} \leq-\frac{\left(H+N^{\prime} \cdot f\right)^{2}}{h^{2}}$. Here we used that $r$ and $f \cdot c_{1}(E)$ are coprime which implies $A$. $f \neq 0$. Our choice of $N^{\prime} \geq N_{0}$ yields $\left(H+N^{\prime} \cdot f\right)^{2}>$ $2 N_{0} H . f$. We conclude that $\frac{r^{2} \Delta(E)}{4}>-\frac{(H+N \cdot f)^{2}}{h^{2}}$. This means that the above two inequalities for $A^{2}$ cannot be satisfied simultaneously and proves the Lemma.

Now $(1) \Longrightarrow(2)$ and $(3) \Longrightarrow(4)$ are obvious, while $(2) \Longrightarrow(1)$ and $(4) \Longrightarrow(3)$ follow immediately from Lemma 2.8.

$(3) \Longrightarrow(2)$. Suppose that $E$ is stable with respect to $H+N_{0} \cdot f$ and unstable with respect to $H+N \cdot f$. Then there exists a $N^{\prime} \in\left(N_{0}, N\right)$ such that $E$ is strictly semistable with respect to $H+N^{\prime} \cdot f$. This contradicts Lemma 2.8. We prove $(1) \Longrightarrow(4)$ following the same pattern.

$(1) \Longrightarrow(5)$. We denote the fiber over $c \in C$ by $X_{c}$. If the restriction $E_{c}:=\left.E\right|_{X_{c}}$ is unstable for the general $c \in C$, then it is unstable for the generic fiber $X_{\eta}$. Let $E_{\eta} \rightarrow E_{\eta}^{\prime \prime}$ be a destabilizing surjection for the generic fiber with $E_{\eta}^{\prime \prime}$ of rank $r^{\prime \prime}$ and degree $d^{\prime \prime}$. This is equivalent to a morphisms of $C$-schemes $\eta \rightarrow \operatorname{Quot}_{E / X / C}^{r^{\prime \prime}, d^{\prime \prime}}$. Since the Quot scheme is projective and $C$ is a smooth curve this morphism extends uniquely to a morphism $C \rightarrow \operatorname{Quot}_{E / X / C}^{r^{\prime \prime}, d^{\prime \prime}}$ which is equivalent to a surjection $\beta: E \rightarrow \tilde{E}^{\prime \prime}$ which extends the surjection on the generic fiber and is flat over $C$. When denoting the saturation of the kernel of $\beta$ by $E^{\prime}$ and and the cokernel of $E^{\prime} \rightarrow E$ by $E^{\prime \prime}$ we obtain a 
short exact sequence $0 \rightarrow E^{\prime} \rightarrow E \rightarrow E^{\prime \prime} \rightarrow 0$ such that for almost all $c \in C$ we have a destabilizing sub-bundle $E^{\prime}{ }_{c} \subset E_{c}$. Setting $A:=\operatorname{rk}\left(E^{\prime}\right) c_{1}(E)-r c_{1}\left(E^{\prime}\right)$ we have therefore $f . A<0$. Then for $\tilde{N} \gg N$ the subsheaf $E^{\prime}$ is destabilizing with respect to $H+\tilde{N} \cdot f$. As before we deduce the existence of an $N^{\prime} \in(N, \tilde{N})$ such that $E$ is strictly semistable with respect to $H+N^{\prime} \cdot f$. This contradicts Lemma 2.8. For the same reasons, $(5) \Longrightarrow(1)$.

Definition 2.9. Fix Chern classes $c_{t}=\left(r, c_{1}, c_{2}\right)$ such that $c_{1} . f$ and $r$ are coprime. A polarization $H$ on $X$ is almost $c_{t}$-fiberlike if for all torsion free coherent sheaves $E$ with Chern classes $c_{t}$ we have

$$
E \text { is stable } \Longleftrightarrow E \text { is semistable }\left.\Longleftrightarrow E\right|_{X_{c}} \text { is stable for a point } c \in C \text {. }
$$

Notice that, since stability is an open condition, the latter condition is equivalent to the stability of $\left.E\right|_{X_{c}}$ for a general point $c \in C$. The existence of an almost $c_{t^{-}}$ fiberlike polarization is shown in Proposition 2.7.

2.3. The orthogonality condition. We denote the genus of $f$ by $g$. For any closed point $c \in C$, write $X_{c}$ for the fiber of $\pi$ over $c$, and $\iota_{c}: X_{c} \rightarrow X$ for the embedding of $X_{c}$ into $X$. Fix Chern classes $c_{t}$ and an almost $c_{t}$-fiberlike polarization $H$ on $X$. We construct a family of vector bundles such that any torsion free coherent sheaf of Chern classes $c_{t}$ is orthogonal to some bundle of the family if and only if it is stable with respect to the fixed polarization.

Recall first that the stability of coherent sheaves on smooth projective curves is equivalent to the existence of orthogonal sheaves.

THEOREM 2.10. (cf. [12, Theorem 5.3]) For a smooth projective curve $Y$ of genus $g$ and a coherent sheaf $E$ on $Y$ of rank $r>0$ and degree $d$ we have equivalent conditions

1. E is a semistable vector bundle,

2. there exist a sheaf $F \neq 0$ such that $\operatorname{Ext}^{i}(F, E)=0$ for all $i \in \mathbb{Z}$, and

3. for any line bundle $L$ of degree $r^{2}\left(\frac{d}{r}-(g-1)\right)$ there exists a vector bundle $F$ of $\operatorname{rank} r^{2}$ and $\operatorname{det}(F) \cong L$ such that $\operatorname{Ext}^{i}(F, E)=0$ for all $i \in \mathbb{Z}$.

REMARK 2.11. If in the above Theorem the genus of $Y$ is one, then the sheaf $F$ in (2) can be chosen of rank $r$ and degree $d$. This is a consequence of Atiyah's classification [1] of vector bundles on elliptic curves.

DEFINITION 2.12. We define the orthogonality condition $(r, d)^{\perp}$ for objects $E \in$ $\mathrm{D}^{\mathrm{b}}(X)$ of the bounded derived category of $X$ as follows:

$$
E \in \mathrm{D}^{\mathrm{b}}(X) \text { is }(r, d)^{\perp} \stackrel{\text { Def }}{\Longleftrightarrow}\left\{\begin{array}{l}
\text { there exists a point } c \in C \text { and a coherent sheaf } \\
F \text { on } X_{c} \text { with } \mathrm{rk}(F)=r, \operatorname{deg}(F)=d \text { such that } \\
\operatorname{Hom}\left(\iota_{c *} F, E[j]\right)=0 \text { for all } j \in \mathbb{Z} .
\end{array}\right\}
$$

For a coherent torsion free sheaf $E$ the orthogonality condition $(r, d)^{\perp}$ is by Theorem 2.10 equivalent to the stability of $E$ restricted to a general fiber of $\pi$. By Proposition 2.7, this is equivalent to the stability of $E$. Resuming, we obtain the following Proposition.

Proposition 2.13. For a torsion free sheaf $E$ with Chern classes $c_{t}$ and an almost $c_{t}$-fiberlike polarization $H$, we have an equivalence 
1. $E$ is $\left(r^{2}, r^{2}\left(\frac{d}{r}-(g-1)\right)\right)^{\perp}$,

2. the restriction of $E$ to the general fiber of $\pi$ is stable,

3. $E$ is slope stable with respect to $H$, and

4. $E$ is slope semistable with respect to $H$.

REMARK 2.14. For an elliptic fibration $\pi: X \rightarrow C$ we can replace by Remark 2.11 the orthogonality condition (1) in the above Proposition by $(r, d)^{\perp}$.

\subsection{A cohomological description of purity.}

Proposition 2.15. Let $E$ be a coherent sheaf on $X$ with Chern classes $c_{t}$. Suppose that $E$ is $\left(r^{2}, r^{2}\left(\frac{d}{r}-(g-1)\right)\right)^{\perp}$. Then there exists a line bundle $L$ on $X$ such that we have an equivalence

$$
\operatorname{Hom}(L, E)=0 \Longleftrightarrow E \text { is torsion free. }
$$

Proof. We take an integer $n$ such that $n(H . f)<1-g+\frac{d}{r}+\frac{\Delta(E)}{2 r}$ holds. After fixing such an integer $n$ we choose $m \in \mathbb{Z}$ such that $\mu\left(\mathcal{O}_{X}(n H+m f)\right)>\mu(E)$ is satisfied. Set now $L=\mathcal{O}_{X}(n H+m f)$.

"£" The line bundle $L$ is a line bundle of slope $\mu(L)>\mu(E)$. Hence if $E$ is torsion free, then $E$ is stable by Proposition 2.13. Whence we deduce $\operatorname{Hom}(L, E)=0$.

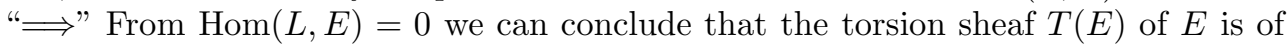
pure dimension one. Since $E$ satisfies the orthogonality condition $\left(r^{2}, r^{2}\left(\frac{d}{r}-(g-1)\right)\right)^{\perp}$, we see that $T(E)$ is supported on fibers of $\pi$. Thus we have $\operatorname{ch}(T(E))=a[f]+b[\mathrm{pt}]$ in $H^{*}(X, \mathbb{Z})$ with $a \geq 0$. If $a=0$ we are done. So we suppose $a \geq 1$. Considering the short exact sequence

$$
0 \rightarrow T(E) \rightarrow E \rightarrow \bar{E} \rightarrow 0
$$

we see that $\bar{E}$ also satisfies the orthogonality condition $\left(r^{2}, r^{2}\left(\frac{d}{r}-(g-1)\right)\right)^{\perp}$, is torsion free, and of discriminant

$$
\Delta(\bar{E})=\Delta(E)+2(a d-b r) .
$$

Since $\bar{E}$ fulfills the orthogonality condition, it is stable for the polarization $H+N \cdot F$ for $N \gg 0$ by Proposition 2.7. So Bogomolov's inequality gives $\Delta(\bar{E}) \leq 0$. We conclude $b \geq \frac{\Delta(E)+2 a d}{2 r}$. So

$$
\mu(T(E)):=\frac{b}{a} \geq \frac{\Delta(E)}{2 a r}+\frac{d}{r} \geq \frac{\Delta(E)}{2 r}+\frac{d}{r} .
$$

The one dimensional sheaf $T(E) \otimes L^{\vee}$ has Euler characteristic $\chi\left(T(E) \otimes L^{\vee}\right)=$ $a(-L . F+\mu(T(E))+1-g)$. The above choice of $L$ and the inequality for $\mu(T(E))$ imply that $\chi\left(T(E) \otimes L^{\vee}\right)>0$ for $a>0$. Thus, we have $a=0$ iff $\operatorname{Hom}(L, T(E))=$ $H^{0}\left(T(E) \otimes L^{\vee}\right)=0$. Since $\operatorname{Hom}(L, E)=0$ implies $\operatorname{Hom}(L, T(E))=0$, we are done.

2.5. A $P$-datum for fibered surfaces. If $E$ is a stable sheaf of given Hilbert polynomial, then there exists an integer $N_{0}$ such that $H^{i}(E(N))=0$ for all $i>0$ and $N \geq N_{0}$. The number $N_{0}$ depends only on the numerical invariants of $E$. In [7, Proposition 5] it is shown that for any Hilbert polynomial $p$ there exists a finite 
number of vector bundles $\left\{L_{i}\right\}_{i=0, \ldots, M}$ and natural numbers $N_{i}$ such that we have an implication for all objects $E \in \mathrm{D}^{\mathrm{b}}(X)$

$$
\left(\operatorname{hom}\left(L_{i}, E[j]\right)=\left\{\begin{array}{ll}
N_{i} & \text { for } j=0 \\
0 & \text { otherwise }
\end{array}\right) \Longrightarrow E \text { is a coherent sheaf of Hilbert polynomial } p .\right.
$$

Using this and Propositions 2.13 and 2.15, allows us to formulate semistability in a homological way:

REMARK 2.16. Let $\pi: X \rightarrow C$ be a fibration of a smooth projective surface over a curve, $f$ a general fiber of $\pi, g$ the genus of $f, c_{t}=\left(r, c_{1}, c_{2}\right)$ Chern classes, with $r$ coprime to $d:=f . c_{1}$. Then there exists a polarization $H$ on $X$, objects $L, L_{i} \in \mathrm{D}^{\mathrm{b}}(X)$, and integers $N_{i}$ such that: an object $E \in \mathrm{D}^{\mathrm{b}}(X)$ satisfies the conditions

$$
\begin{aligned}
& \text { (h) } \operatorname{hom}\left(L_{i}, E[j]\right)= \begin{cases}N_{i} & \text { for } j=0 \\
0 & \text { otherwise, }\end{cases} \\
& \text { (tf) } \operatorname{Hom}(L, E)=0, \text { and } \\
& \text { (o) } E \text { is }\left(r^{2}, r^{2}\left(\frac{d}{r}-(g-1)\right)\right)^{\perp}
\end{aligned}
$$

if and only if $E$ is a semistable sheaf of Chern classes $c_{t}$. Indeed the conditions (h) and (tf) ensures that $E$ is a torsion free sheaf and then we can apply Proposition 2.13 . For an elliptic fibration $\pi: X \rightarrow C$ we can replace by Remark 2.14 the orthogonality condition (o) in the above theorem by $(r, d)^{\perp}$.

2.6. Exceptional fibers. In this final subsection, we analyze, given a semistable sheaf $E$ of fixed Chern classes, how unstable its restriction to a fiber can be. Here we assume that the canonical class $K_{X}$ is trivial.

Proposition 2.17. Let $K_{X}$ be trivial, and $E$ be a vector bundle on $X$ with Chern classes $c_{t}=\left(r, c_{1}, c_{2}\right)$. We assume that the rank $r$ and the fiber degree $c_{1} . f$ are coprime, and $E$ is stable for an almost $c_{t}$-fiberlike polarization $H$. We set $t:=$ $\frac{1}{2} h^{1}(\mathcal{E} n d(E))=1-\frac{1}{2} \chi(\mathcal{E} n d(E))$.

1. Take a fiber $X_{0}$, denote $E_{0}:=\left.E\right|_{X_{0}}$, and consider a surjection $E_{0} \stackrel{\alpha}{\rightarrow} Q$ with $Q$ a sheaf on $X_{0}$ of positive rank $r_{Q}$. We write $E_{0}^{\prime}$ for $\operatorname{ker}(\alpha)$ which is a vector bundle on $X_{0}$ and $r^{\prime}$ for its rank (remark that $r^{\prime}=r-r_{Q}$ ). Then we have the inequality

$$
r r^{\prime}\left(\mu\left(E_{0}^{\prime}\right)-\mu\left(E_{0}\right)\right)=r r_{Q}\left(\mu\left(E_{0}\right)-\mu(Q)\right) \leq t .
$$

2. If the restriction $E_{i}$ of $E$ to the fibers $X_{i}$ is not semistable, then we have short exact sequences $0 \rightarrow E_{i}^{\prime} \rightarrow E_{i} \rightarrow E_{i}^{\prime \prime} \rightarrow 0$ of $X_{i}$-vector bundles. Denote by $r_{i}^{\prime}$ and $r_{i}^{\prime \prime}$ the ranks of $E_{i}^{\prime}$ and $E_{i}^{\prime \prime}$ respectively. We have an inequality

$$
\sum_{i=1}^{l} r r_{i}^{\prime}\left(\mu\left(E_{i}^{\prime}\right)-\mu\left(E_{i}\right)\right)=\sum_{i=1}^{l} r r_{i}^{\prime \prime}\left(\mu\left(E_{i}\right)-\mu\left(E_{i}^{\prime \prime}\right)\right) \leq t .
$$

3. The number of fibers $X_{i}$ where the restriction $E_{i}$ of $E$ to $X_{i}$ is not stable cannot exceed $t$.

Proof. (1) We consider the composition of surjections $E \rightarrow E_{0} \rightarrow Q$ and denote it kernel by $E^{\prime}$. On a general fiber, the restriction of $E^{\prime}$ coincides with the restriction of $E$. So we know that $E^{\prime}$ is stable for some almost-fiberlike polarization. This implies $h^{0}\left(\mathcal{E} n d\left(E^{\prime}\right)\right)=h^{2}\left(\mathcal{E} n d\left(E^{\prime}\right)\right)=1$. Thus, we have

$$
\chi\left(\mathcal{E} n d\left(E^{\prime}\right)\right)-\chi(\mathcal{E} n d(E))=2 t-h^{1}\left(\mathcal{E} n d\left(E^{\prime}\right)\right) \leq 2 t .
$$


Using the Riemann-Roch theorem to compute $\chi\left(\mathcal{E} n d\left(E^{\prime}\right)\right)-\chi(\mathcal{E} n d(E))$ we obtain

$\chi\left(\mathcal{E} n d\left(E^{\prime}\right)\right)-\chi(\mathcal{E} n d(E))=\left(\operatorname{ch}\left(\mathcal{E} n d\left(E^{\prime}\right)\right)-\operatorname{ch}(\mathcal{E} n d(E))\right) \operatorname{td}(X)=2 r_{Q} \operatorname{deg}\left(E_{0}\right)-2 r \operatorname{deg}(Q)$.

Together with the above inequality this yields the inequality in the statement (1). To check the equality $r r^{\prime}\left(\mu\left(E_{0}^{\prime}\right)-\mu\left(E_{0}\right)\right)=\operatorname{rr}_{Q}\left(\mu\left(E_{0}\right)-\mu(Q)\right)$ is straightforward.

(2) Is shown analogously to (1) by considering $E^{\prime}=\operatorname{ker}\left(E \rightarrow \bigoplus_{i=1}^{l} E_{i}^{\prime \prime}\right)$.

(3) Follows from (2) since for destabilizing $E_{i}^{\prime} \subset E_{i}$, each individual summand in the above sum is a positive integer.

3. Isomorphisms between moduli spaces and the EFM-algorithm. Let $\pi: X \rightarrow \mathbb{P}^{1}$ be a K3 elliptic surface with at most finitely many singular fibers admitting a section, and $\widehat{X} \rightarrow \mathbb{P}^{1}$ its relative Jacobian. We have a Fourier-Mukai equivalence $\Psi$ between $\mathrm{D}^{\mathrm{b}}(X)$ and $\mathrm{D}^{\mathrm{b}}(\widehat{X})$ whose kernel is the relative Poincaré bundle. Given a Mukai vector $v$ such that the moduli space $M_{X}(v)$ is smooth projective irreducible and non-empty, we describe under which conditions $\Psi$ induces an isomorphism between $M_{X}(v)$ and $M_{\widehat{X}}(\hat{v})$, where $\hat{v}$ depends only on $v$.

In order to do this, we use the orthogonality from Proposition 2.13 which guarantees the semistability of torsion free sheaves with Mukai vector $v$. In particular, this orthogonality condition is preserved (under natural numerical modifications) by the Fourier-Mukai equivalence. For a stable sheaf $E$ in $M_{X}(v)$, it is then enough to check that $\Psi(E)$ is, up to a shift, a torsion free sheaf to get that it is stable with Mukai vector $\hat{v}$.

Notice that we restrict to the K3 case in order to make all numerical calculation clearer. However, the arguments can be extended to a general elliptic fibration with at most finitely many nodal fibers over a smooth projective curve, provided that the numerical data are properly adapted.

Let $f$ denote the class of a general fiber of $\pi$. If $E$ is a torsion free sheaf on $X$, we call the integer $c_{1}(E) . f$ its fiber degree. If $E$ is an object in $\mathrm{D}^{\mathrm{b}}(X)$, then we define the fiber degree (resp. the rank) of $E$ by the alternate sum of the fiber degrees (resp. the ranks) of its cohomology sheaves.

Let us fix a Mukai vector $v=(r, \Lambda, c)$, such that $\Lambda . f$ and $r$ are coprime. Up to twisting with a line bundle, we can uniquely fix $\Lambda$. $f=-d$ with $0<d<r$. Let us fix an almost $v$-fiberlike polarization $H$. Then the moduli space of semistable torsion free sheaves with Mukai vector $v$ on $X$ is an irreducible smooth projective variety of dimension $2 t$, where $2-2 t=\langle v, v\rangle$. We will denote this moduli space by $M_{X}(v)$.

Let $\widehat{X}$ be the relative Jacobian of $X$, that is the elliptic surface over $\mathbb{P}^{1}$, whose fiber over a point $c$ is the Jacobian of the elliptic curve $X_{c}$. The surface $\widehat{X}$ can be described as a relative moduli space over $\mathbb{P}^{1}$, for definition and properties we refer to [8], in particular Theorem 4.3.7. Notice that the existence of a section of $\pi$ implies that $\widehat{X}$ and $X$ are isomorphic.

Let $\mathcal{P}$ be the universal sheaf over $X \times \widehat{X}$. We consider the Fourier-Mukai transform $\Psi: \mathrm{D}^{\mathrm{b}}(X) \rightarrow \mathrm{D}^{\mathrm{b}}(\widehat{X})$ with kernel $\mathcal{P}$, which is defined as

$$
\Psi(-)=R \hat{p}_{*}\left(p^{*}(-) \otimes \mathcal{P}\right)
$$

where $p$ and $\hat{p}$ denote the projection from $X \times \widehat{X}$ to $X$ and $\widehat{X}$ respectively. It is well known [2] that $\Psi$ is an equivalence between $\mathrm{D}^{\mathrm{b}}(X)$ and $\mathrm{D}^{\mathrm{b}}(\widehat{X})$. We will denote by $\Phi$ the relative quasi-inverse of $\Psi$, that is $\Phi \circ \Psi=[1]$. Denote by $\mathcal{Q}$ the kernel of $\Phi$.

Let $\hat{v}=(d, \hat{\Lambda}, \hat{c})$ be the Mukai vector on $\widehat{X}$ with: 
1. $\hat{\Lambda} \cdot \hat{f}=r$,

2. $\langle\hat{v}, \hat{v}\rangle=\langle v, v\rangle$.

The moduli space $M_{\widehat{X}}(\hat{v})$ is irreducible smooth projective of the same dimension $2 t$ as $M_{X}(v)$.

Fix an almost $v$-fiberlike polarization $H$. Remark 2.16 describes how slopestability is equivalent to a $P$-stability in the derived category $\mathrm{D}^{\mathrm{b}}(X)$. In particular, by Proposition 2.13, a torsion free sheaf with given Hilbert polynomial (and hence Mukai vector $v$ ) is semistable if and only if it satisfies the orthogonality condition (o) of Remark 2.16. The most important feature is that the condition (o) is well behaved with respect to the Fourier-Mukai transform, that is the image under $\Psi$ of any stable sheaf satisfies an orthogonality condition. Then if a sheaf in $M_{X}(v)$ is mapped by $\Psi$ to a torsion free sheaf, this sheaf will be stable with Mukai vector $\hat{v}$, by Prop. 2.13. This allows finally to prove one of the main results of this paper.

THEOREM 3.1. In the previous notations, if $t<r$, the moduli spaces $M_{X}(v)$ and $M_{\widehat{X}}(\hat{v})$ are isomorphic and the isomorphism is induced by the Fourier-Mukai transform $\Psi$.

Before the proof of Theorem 3.1, we give some result on the preservation of stability which are independent on the dimension $2 t$ of the moduli spaces and which follow from the purely homological interpretation of stability given in Remark 2.16. Recall first the general definition of $\mathrm{WIT}_{i}$ and $\mathrm{IT}_{i}$ sheaves.

Definition 3.2. Let $\Pi: \mathrm{D}^{\mathrm{b}}(Y) \rightarrow \mathrm{D}^{\mathrm{b}}(Z)$ be a functor between the derived categories of two smooth projective varieties. A sheaf $E$ on $Y$ is $\Pi-\mathrm{WIT}_{i}$ if $\Pi(E)[i]$ is a sheaf on $Z$, that is an object in $\mathrm{D}^{\mathrm{b}}(Z)$ whose only nontrivial cohomology sheaf lives in degree zero. We denote in this case $\Pi(E)[i]$ by $\widehat{E}$. A $\Pi-\mathrm{WIT}_{i}$ sheaf $E$ is $\Pi-\mathrm{IT}_{i}$ if $\widehat{E}$ is a vector bundle.

Remark that a sheaf $E$ in $\mathrm{D}^{\mathrm{b}}(X)$ is $\Psi$-WIT $i$ if and only if $\Psi(E)$ is $\Phi-\mathrm{WIT}_{1-i}$, just because $\Phi \circ \Psi=[1]$. We have the following results by [2].

Lemma 3.3. Let $E$ be an object in $\mathrm{D}^{\mathrm{b}}(X)$ of rank $a$ and fiber degree $b$, then $\Psi(E)$ has rank $-b$ and fiber degree $a$.

Let $E$ be a semistable torsion free sheaf on $X$ with negative slope. Then $E$ is $\Psi-W I T_{1}$.

Proof. These are special cases of [2, Theorem 5.3 and Lemma 6.4], once remarked that the matrix associated to $\Psi$ is

$$
\left(\begin{array}{cc}
0 & 1 \\
-1 & 0
\end{array}\right)
$$

For the convenience of the reader we include the following table which shows the effect of $\Psi$ applied to sheaves supported on fibers. To do so we introduce the shorthand $T_{a, b}$ for a rank $a$ vector bundle of degree $b$ on a fiber of $X \rightarrow C$ considered as a sheaf on $X$. 


\begin{tabular}{|c|c|c|c|c|}
\hline object & description & $\Psi$ (object) & & description \\
\hline$T_{a, b}, b>0$ & stable bundle on a fiber & $T_{b,-a}$ & & stable bundle on a fiber \\
\hline$T_{a, b}, b<0$ & stable bundle on a fiber & $T_{-b, a}[-1]$ & & $\begin{array}{l}\text { stable bundle on a fiber, } \\
\text { shifted to degree } 1\end{array}$ \\
\hline $\begin{array}{l}k(x) \quad= \\
T_{0,1}\end{array}$ & skyscraper sheaf of $x$ & $\mathcal{P}_{x}=T_{1,0}$ & & $\begin{array}{l}\text { line bundle of degree } 0 \\
\text { on a fiber }\end{array}$ \\
\hline $\mathcal{P}_{x}=T_{1,0}$ & $\begin{array}{l}\text { line bundle of degree } 0 \\
\text { on a fiber }\end{array}$ & $\begin{array}{l}k(x)[-1] \\
T_{0,1}[-1]\end{array}$ & $=$ & $\begin{array}{l}\text { skyscraper sheaf, shifted } \\
\text { to degree } 1\end{array}$ \\
\hline
\end{tabular}

The most important feature of the orthogonality condition from Proposition 2.13 is that it is well behaved under relative Fourier-Mukai equivalences.

Lemma 3.4. For any $m$ and $n$ in $\mathbb{Z}$, an object $A$ in $\mathrm{D}^{\mathrm{b}}(X)$ is $(m, n)^{\perp}$ if and only if $\Psi(A)$ is $(n,-m)^{\perp}$. 3.3.

Proof. Since simple objects on fibers are shifted sheaves, this follows from Lemma

REMARK 3.5. Let $Y:=J_{X}(a, b)$ and consider the relative Fourier-Mukai equivalence $\Pi: \mathrm{D}^{\mathrm{b}}(X) \rightarrow \mathrm{D}^{\mathrm{b}}(Y)$ with the associated matrix $P$ as described by Bridgeland [2]. Then the same proof of Lemma 3.4 shows that $A$ in $\mathrm{D}^{\mathrm{b}}(X)$ is $(m, n)^{\perp}$ if and only if $\Pi(A)$ is $\left(\left(P \cdot\left(\begin{array}{c}m \\ n\end{array}\right)\right)^{t}\right)^{\perp}$ in $\mathrm{D}^{\mathrm{b}}(Y)$.

Corollary 3.6. Let $E$ be a stable sheaf in $M_{X}(v)$. Then $E$ is $\Psi-W I T_{1}$ and the sheaf $\widehat{E}$ is $(d, r)^{\perp}$.

Proof. The slope of $E$ is $-d / r<0$ and $E$ is torsion free, then it is $\Psi-\mathrm{WIT}_{1}$ by Lemma 3.3. Now $\Psi(E)$ is $(-d,-r)^{\perp}$ by Lemma 3.4, then the proof follows from $\widehat{E}=\Psi(E)[1]$.

The last step is given now by determining conditions on $E$ to have $\widehat{E}$ torsion free.

Proposition 3.7. Let $E$ be a stable sheaf in $M_{X}(v)$. The sheaf $\widehat{E}$ is torsion free if and only if $E$ is a vector bundle such that for any fiber $F$ of $\pi$, the restriction $E_{F}$ does not admit a sub-bundle of positive slope.

Proof. Suppose first that $E$ is not locally free and consider the sequence

$$
0 \longrightarrow E \longrightarrow E^{\vee \vee} \longrightarrow \tau \longrightarrow 0 \text {. }
$$

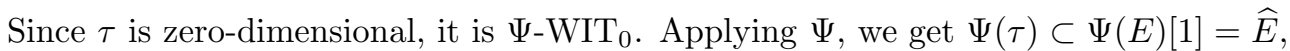
which shows that $\widehat{E}$ is not torsion free.

Now let $E$ be a vector bundle. Suppose that the sheaf $\widehat{E}$ has a zero dimensional torsion, that is, there exists a point $p$ of $\widehat{X}$ such that $k(p) \subset \widehat{E}$, and let $\widehat{X}_{0}$ be the fiber of $\hat{\pi}$ containing $p$. Then for any line bundle $L_{0}$ in $\operatorname{Pic}^{0}\left(\widehat{X}_{0}\right)$, there is a nontrivial morphism $i_{*} L_{0} \rightarrow \widehat{E}$, where $i$ is the embedding of $\widehat{X}_{0}$ in $\widehat{X}$. Equivalently, for all points $x$ in $X_{0}$, there is a nontrivial morphism $\Psi(k(x)) \rightarrow \widehat{E}=\Psi(E)[1]$. Since $\Psi$ is an equivalence, we get that for all points $x$ in the fiber $X_{0}$, the extension group $\operatorname{Ext}^{1}(k(x), E)$ is nontrivial, which contradicts the locally freeness of $E$.

If $T$ is a non-zero torsion subsheaf of $\widehat{E}$, it is then pure of dimension 1. Moreover, it has to be supported on a finite number of fibers of $\hat{\pi}$. Indeed, if $\widehat{X}_{0}$ is a fiber such that $\widehat{X}_{0} \cap \operatorname{Supp} T$ is a finite set of points, then the restriction of $E$ to $X_{0}$ is unstable, and this can happen only for finitely many fibers. 
If $\widehat{X}_{0}$ is a fiber in the support of $T$ and $T_{0}$ the restriction, then $\mu_{\max }\left(T_{0}\right)<0$, where $\mu_{\max }$ denotes the maximal slope of a subsheaf of $T_{0}$ on the elliptic curve $\widehat{X}_{0}$. Indeed, if $\mu_{\max }\left(T_{0}\right) \geq 0$, we would have a nontrivial morphism $L_{0} \rightarrow T_{0}$ for some $L_{0}$ in $\operatorname{Pic}^{0}\left(\widehat{X}_{0}\right)$ and this would be a contradiction, as $E$ is a vector bundle, as before. Suppose for simplicity that $T$ is pure supported on a single fiber $\widehat{X}_{0}$, then $T$ is $\Phi-\mathrm{WIT}_{1}$. Let $\check{T}:=\Phi(T)[1]$. The sheaf $\check{T}$ is supported on the fiber $X_{0}$ and has positive slope. Then:

$$
\operatorname{Ext}_{X}^{1}(E, \check{T})^{\vee}=\operatorname{Ext}_{X}^{1}(\check{T}, E)=\operatorname{Hom}_{\widehat{X}}(T, \widehat{E}) \neq 0,
$$

where we applied Serre duality at the first step. Since $E$ is locally free,

$$
\operatorname{Ext}_{X}^{1}(E, \check{T})=\operatorname{Ext}_{X_{0}}^{1}\left(E_{0}, \check{T}\right)=\operatorname{Hom}_{X_{0}}\left(\check{T}, E_{0}\right)^{\vee}
$$

where we applied Serre duality on $X_{0}$. Then we get the proof.

REMARK 3.8. As a consequence of Propositions 2.13 and 3.7 we get a birational $\operatorname{map} M_{X}(v) \rightarrow M_{\widehat{X}}(\widehat{v})$.

Proof of Theorem 3.1. To prove Theorem 3.1, we apply Prop. 2.13. Recall that, by Cor. 3.6, the sheaf $\widehat{E}$ is $(d, r)^{\perp}$. Then the semistability of $\widehat{E}$ is ensured by Prop. 2.13 , if we show that, for all $E$ in $M_{X}(v)$, the sheaf $\widehat{E}$ is torsion free. To do that, we have to check both conditions from Proposition 3.7.

Lemma 3.9. Let $E$ be a vector bundle on $X$ of Mukai vector $v$. If $t<r+d$, the restriction of $E$ to any fiber of $\pi: X \rightarrow \mathbb{P}^{1}$ does not admit a sub-bundle of positive slope.

Proof. Suppose $E$ is a vector bundle and $B$ a sheaf supported on a fiber $X_{0}$ with positive slope. As a sheaf on $X_{0}, B$ has strictly positive rank and degree, say $r_{0}$ and $d_{0}$ respectively. Then part (1) of Proposition 2.17 gives $r d_{0}+r_{0} d \leq t$. Since $r_{0}, d_{0} \geq 1$, this cannot happen for $t<r+d$.

Lemma 3.10. Let $E$ be a semistable sheaf on $X$ of Mukai vector $v$. If $t<r$, then $E$ is a vector bundle.

Proof. Suppose that $E$ is not a vector bundle. Then there is a length $l$ zerodimensional torsion sheaf $T$ and an exact sequence

$$
0 \longrightarrow E \longrightarrow E^{\vee \vee} \longrightarrow T \longrightarrow 0 \text {. }
$$

The Mukai vector of $E^{\vee \vee}$ is $v^{\prime}=(r, \Lambda, c+l)$ and $E^{\vee \vee}$ is semistable. The proof follows by checking that for $t<r$ the moduli space $M_{X}\left(v^{\prime}\right)$ is empty.

By Lemmas 3.9 and 3.10, if $t<r$, we get that for all $E$ in $M_{X}(v)$ the sheaf $\widehat{E}=\Psi(E)[1]$ is semistable of Mukai vector $\hat{v}$.

Consider the equivalence $\Phi: \mathrm{D}^{\mathrm{b}}(\widehat{X}) \rightarrow \mathrm{D}^{\mathrm{b}}(X)$, the relative quasi inverse of $\Psi$. We have $\Psi \circ \Phi=[1]$. Recall that the varieties $M_{X}(v)$ and $M_{\widehat{X}}(\hat{v})$ are smooth, projective, simply connected of the same dimension $2 t$. Since $\Phi$ and $\Psi$ are equivalences and each other quasi inverse (up to a shift), $\Phi$ also preserves stability, that is $\Phi(F)$ is a stable sheaf with Mukai vector $v$ for all $F$ in $M_{\widehat{X}}(\hat{v})$. Moreover, we have $\operatorname{Ext}^{1}(F, F)=$ $\operatorname{Ext}^{1}(\Phi(F), \Phi(F))$. Therefore the morphisms induced by $\Phi$ and $\Psi$ are étale. We 
finally ended up with an étale bijective morphism between smooth projective simply connected varieties. Thus, $\Psi$ induces an isomorphism ${ }^{1}$.

REMARK 3.11. Remark that, if we stick to the case $r=2$ described by Friedman [4], Theorem 3.1 gives an isomorphism between the moduli space and the Hilbert scheme $\operatorname{Hilb}^{t}(X)$ of length $t$ quotients of $\mathcal{O}_{X}$ only for $t=1$. The case $t=r=2$, in which Friedman showed the existence of an isomorphism, will be described in the next Section.

3.1. The Euclid-Fourier-Mukai algorithm. In the previous subsection we have shown how, under a condition on the dimension of the moduli space, the FourierMukai transform $\Psi$ induces an isomorphism between $M_{X}(v)$ and $M_{\widehat{X}}(\hat{v})$. The very special choice of $\widehat{X}$ could look more restrictive than the construction performed by Bridgeland in [2]. We show that it is indeed enough to consider, given an elliptic surface $X$, the Jacobian $\widehat{X}$ and perform a finite number of steps to get a more general description.

Given coprime integers $r$ and $d$, with $0<d<r$, we can consider the Euclidean algorithm applied to the pair $(r,-d)$ : its elementary step is given by sending $(r,-d)$ to $(d,-s)$, with $-s \equiv r$ modulo $d$ and $0<s<d$. Since $r$ and $d$ are coprime, the algorithm will end with the pair $(1,0)$.

The Mukai vector $v=(r, \Lambda, c)$ on $X$ gives the integers $r,-d$ and $t$, where $-d$ is the fiber degree of $\Lambda$ and $2 t$ the expected dimension of the moduli space. Recall that $r$ and $d$ are coprime, $r \geq 2$ and $0<d<r$. Consider the Jacobian $\widehat{X}$ of $X$ and the Fourier-Mukai equivalence $\Psi: \mathrm{D}^{\mathrm{b}}(X) \rightarrow \mathrm{D}^{\mathrm{b}}(\widehat{X})$. The functor $\Psi[1]$ sends sheaves with a Mukai vector $v$ on $X$ to sheaves with a Mukai vector $\hat{v}$ on $\widehat{X}$. The integer $t$ is invariant under this operation, while the pair $(r,-d)$ is sent to the pair $(d, r)$. We can fix a line bundle $L$ on $\widehat{X}$ such that, for all $E$ in $M_{\widehat{X}}(\hat{v})$, the sheaf $E \otimes L$ has fiber degree $-s$, where $-s \equiv r$ modulo $d$ and $0<s<d$. In particular, $s$ and $d$ are coprime. This last operation gives rise to a natural isomorphism between $M_{\widehat{X}}(\hat{v})$ and $M_{\widehat{X}}(\hat{w})$, where $\hat{w}$ is the Mukai vector of $E \otimes L$.

We can now define the Euclid-Fourier-Mukai algorithm as follows: given a Mukai vector $v=(r, \Lambda, c)$ as before, consider the integers $t$ (half dimension of the moduli space), $r$ (rank) and $-d$ (fiber degree), with $r$ and $-d$ coprime and $0<d<r$. Consider the Fourier-Mukai transform $\Psi$ : it takes (up to a shift and a tensor by a line bundle) the pair $(r,-d)$ to the pair $(d,-s)$, leaving $t$ invariant. If $d=1$ and $s=0$, we stop. If $0<s<d$, we continue. The Euclid-Fourier-Mukai algorithm is then obtained by applying this procedure to the moduli space $M_{\widehat{X}}(\hat{v})$. Remark that the algorithm always ends with the Mukai vector $(1,0, t)$.

As a consequence, starting from the moduli space $M_{X}(v)$, the Euclid-FourierMukai algorithm ends up with the Hilbert scheme $\operatorname{Hilb}^{t}(X)$ in a finite number of steps. Indeed remember that, since $\pi: X \rightarrow \mathbb{P}^{1}$ admits a section, $X$ is isomorphic to its Jacobian $\widehat{X}$.

Since the rank is strictly decreasing and $t$ is constant, the condition given by Theorem 3.1 gets more strict at every step. Consider the Mukai vector $v$, with $t$ and $(r,-d)$ as before and the Euclid-Fourier-Mukai algorithm. The last step has the form $(l,-1) \rightarrow(1,0)$ for $l \geq 2$ integer.

Corollary 3.12. If $t<l$, the moduli space $M_{X}(v)$ is isomorphic to $\operatorname{Hilb}^{t}(X)$.

\footnotetext{
${ }^{1}$ This argument shortens a previous proof and was suggested to us by the anonymous referee. We acknowledge him/her for that.
} 
Given the pair $(r,-d)$, there exist integers $a$ and $b$ such that $b r+a d=1$, and we can fix $a$ uniquely such that $0<a<r$. We have the following result by Bridgeland.

TheOREM 3.13. If $t<r / a$, the moduli space $M_{X}(v)$ is isomorphic to $\mathrm{Hilb}^{t}(X)$.

Proof. [2, Lemma 7.4]

Corollary 3.12 and Theorem 3.13 give indeed the same result.

Lemma 3.14. With the previous notations, $l=\lceil r / a\rceil$.

We will give the proof of this rather technical Lemma in the Appendix, see Lemma A.1.

3.2. Isomorphisms between Picard groups. Consider any Mukai vector $v$ with rank and fiber degree coprime. The existence of a birational map $M_{X}(v) \rightarrow$ $M_{\widehat{X}}(\hat{v})$ (see Remark 3.8) and the Euclid-Fourier-Mukai algorithm give, in the case $X$ is a K3 surface, a chain of isomorphisms of the Picard groups of the moduli spaces, ending with the Picard group of the Hilbert scheme of the given dimension. phism

Corollary 3.15. The birational map $\varepsilon: M_{X}(v) \rightarrow M_{\widehat{X}}(\hat{v})$ induces an isomor-

$$
\operatorname{Pic}\left(M_{X}(v)\right) \simeq \operatorname{Pic}\left(M_{\widehat{X}}(\hat{v})\right) .
$$

Proof. Recall that both $M_{X}(v)$ and $M_{\widehat{X}}(\hat{v})$ are irreducible holomorphic symplectic varieties. By [10, Prop. 1.6.2], given any birational map $\phi: M_{1} \rightarrow M_{2}$ between irreducible holomorphic symplectic manifolds, there are closed subsets $Z_{1} \subset M_{1}$ and $Z_{2} \subset M_{2}$, both of codimension at least 2 , such that $\phi$ gives an isomorphism $M_{1} \backslash Z_{1} \simeq$ $M_{2} \backslash Z_{2}$ and then an isomorphism $\operatorname{Pic}\left(M_{1}\right) \simeq \operatorname{Pic}\left(M_{2}\right)$. We get than the statement as a consequence of Remark $3.8 .^{2} \square$

Corollary 3.16. Let $v=(r, \Lambda, c)$ be a Mukai vector on $X$ with $\Lambda . f=-d$, with $r, d$ coprime, and $d$ any integer. Then we have an isomorphism

$$
\operatorname{Pic}\left(M_{X}(v)\right) \simeq \operatorname{Pic}\left(\operatorname{Hilb}^{t}(X)\right),
$$

where $2 t$ is the dimension of the moduli space $M_{X}(v)$.

Proof. By tensoring with a line bundle, we can suppose, up to isomorphism of $M_{X}(v)$, that $0<d<r$. The proof follows applying recursively Corollary 3.15 to the steps of the Euclid-Fourier-Mukai algorithm and reminding that, since $X \rightarrow \mathbb{P}^{1}$ admits a section, $\widehat{X}$ is ismorphic to $X$.

4. Birational transformations between the moduli spaces. As in the previous Section, let $\pi: X \rightarrow \mathbb{P}^{1}$ be a K3 elliptic fibration with at most nodal singular fibers and a section, and $\widehat{X} \rightarrow \mathbb{P}^{1}$ be the relative Jacobian. If $t<r$, we can establish an isomorphism between $M_{X}(v)$ and $M_{\widehat{X}}(\hat{v})$. In this Section, we deal with $t \geq r$ and we describe the birational correspondence between the moduli spaces, obtaining a Mukai flop for $r \leq t<r+d$, which is an isomorphism if and only if $r=t=2$. It is also clear that, for any $t \geq r>2$, the map cannot be an isomorphism. For $r=2$, we argue how in the case $t=4$, the conjecture formulated by Friedman should be false. This Section is devoted to the proof of the following Theorem.

\footnotetext{
${ }^{2}$ this argument, which shortens our previous one, was suggested us by the anonymous referee, who is acknowledged for that.
} 
TheOREM 4.1. Let $r \leq t<r+d$. Then the Fourier-Mukai equivalence $\Psi$ induces a Mukai flop between $M_{X}(v)$ and $M_{\widehat{X}}(\hat{v})$, unless $t=r=2$, in which case it induces an isomorphism.

As for the proof of Theorem 3.1, the main ingredient here is given by the identification of the slope stability with orthogonality provided in Proposition 2.13 and in particular by the well-behavior of the orthogonality condition. We will base upon it and results from Section 3 to describe the indeterminacy loci of the birational maps induced by $\Psi$ and $\Phi$.

Proof of Theorem 4.1. First of all, recall Corollary 3.6 which states that all $E$ in $M_{X}(v)$ are $\Psi-\mathrm{WIT}_{1}$ and such that $\widehat{E}$ is $(d, r)^{\perp} . \widehat{E}$ is in $M_{\widehat{X}}(\hat{v})$ whenever it is a torsion free sheaf.

LEMMA 4.2. Let $E$ be a stable sheaf in $M_{X}(v)$ and $t<r+d$. The sheaf $\widehat{E}$ is not torsion free if and only if $E$ is not a vector bundle. Moreover this is the case if and only if there is a point $x$ in $X$ and a short exact sequence

$$
0 \longrightarrow E \longrightarrow E^{\vee \vee} \longrightarrow k(x) \longrightarrow 0 \text {. }
$$

Proof. The first equivalence follows from Proposition 3.7, and Lemma 3.9. For the second one, suppose $E$ is not a vector bundle and we have the exact sequence

$$
0 \longrightarrow E \longrightarrow E^{\vee \vee} \longrightarrow T \longrightarrow 0
$$

where $T$ is a positive length $l$ 0-dimensional torsion sheaf. Arguing as in the proof of Lemma 3.10, since $t<r+d<2 r$, we must have $l=1$, that is $T=k(x)$ for a point $x$ in $X$.

We deduce that $\Psi$ induces a birational map

$$
\varepsilon: M_{X}(v) \rightarrow M_{\widehat{X}}(\hat{v}) \text {. }
$$

Let $E$ be a non locally free sheaf in $M_{X}(v)$ and denote by $u$ the Mukai vector of $E^{\vee \vee}$. We have $u=(r, \Lambda, c+1)$, independently of the point $x$ in the exact sequence (4.1). Remark that $E^{\vee \vee}$ is a stable vector bundle and the moduli space $M_{X}(u)$ has dimension $2 t-2 r$ and is then isomorphic to $M_{\widehat{X}}(\hat{u})$.

Proposition 4.3. The indeterminacy locus of $\varepsilon$ is a $\mathrm{P}^{r-1}$-bundle over $X \times M_{X}(u)$ and naturally isomorphic via $\Psi$ to the a $\mathbb{P}^{r-1}$-bundle over $X \times M_{\widehat{X}}(\hat{u})$ associated to the universal extension of the form $\mathbb{P E x t}^{1}\left(F, \mathcal{P}_{x}\right)$, for $F$ in $M_{\widehat{X}}(\hat{u})$ and $\mathcal{P}_{x}$ in $\widehat{\widehat{X}} \cong X$. In particular, the indeterminacy locus has codimension $r-1$.

Proof. Let $E$ in $M_{X}(v)$ be such that $\Psi(E)$ is not in $M_{\widehat{X}}(\hat{v})$. Then, by Lemma 4.2 , there is a point $x$ in $X$ and an exact sequence

$$
0 \longrightarrow E \longrightarrow E^{\vee \vee} \longrightarrow k(x) \longrightarrow 0 .
$$

Moreover, any element of $M_{X}(u)$ appears in a sequence like (4.2) for some $E$ in $M_{X}(v)$ and some point $x$ in $X$. Apply $\Psi$ to get the exact sequence

$$
0 \longrightarrow \mathcal{P}_{x} \longrightarrow \widehat{E} \longrightarrow \widehat{E^{\vee V}} \longrightarrow 0
$$

of sheaves on $\widehat{X}$. The sheaves $\widehat{E}$ are then parameterized, as $E$ moves in the indeterminacy locus, by extensions of an element of $M_{\widehat{X}}(\hat{u})$ by a degree zero line bundle on 
a fiber of $\hat{\pi}$. Conversely, it is clear that any such extension corresponds to the image of an element of $M_{X}(v)$ whose image under $\Psi$ is not torsion free.

The dimension of the fiber is easily calculated by $\operatorname{Ext}^{1}\left(F, \mathcal{P}_{x}\right) \cong \operatorname{Hom}\left(E^{\vee \vee}, k(x)\right)$. Having computed the dimension of $M_{X}(u)$, the statement about the codimension is straightforward.

Consider the equivalence $\Phi$, the relative quasi inverse of $\Psi$. Then $\Phi$ induces a birational map

$$
\rho: M_{\widehat{X}}(\hat{v}) \rightarrow M_{X}(v)
$$

Once again by the preservation of orthogonality described in Lemma 3.4, we have that, for $F$ in $M_{\widehat{X}}(\hat{v}), \Phi(F)$ is stable whenever it is (eventually up to shift) a torsion free sheaf.

LEMmA 4.4. Let $E$ be a sheaf in $M_{\widehat{X}}(\hat{v})$. Then $E$ is $\Phi-W I T_{0}$ if and only if it is $\Phi-I T_{0}$.

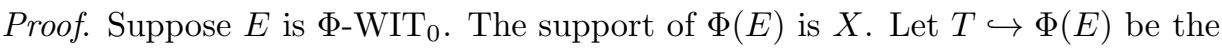
maximal torsion subsheaf. We have the exact sequence

$$
0 \longrightarrow T \longrightarrow \Phi(E) \longrightarrow F \longrightarrow 0
$$

Suppose $T$ has zero dimensional torsion, that is, there exists a point $p$ of $X$ such that $k(p) \subset \Phi(E)$, and let $X_{0}$ be the fiber of $\pi$ containing $p$. Then for any line bundle $L_{0}$ in $\operatorname{Pic}^{0}\left(X_{0}\right)$, there is a nontrivial morphism $i_{*} L_{0} \rightarrow \Phi(E)$, where $i$ is the embedding of $X_{0}$ in $X$. Equivalently, for all point $x$ in $\widehat{X}_{0}$, there is a unique nontrivial morphism $\Phi(k(x))[1] \rightarrow \Phi(E)$. We get then that for all points $x$ in the fiber $\widehat{X}_{0}$, the extension group $\operatorname{Ext}^{1}(E, k(x))$ is nontrivial, which contradicts the locally freeness of $E$. Then $T$ has to be pure of dimension 1 . Moreover, $T$ has to be supported on a finite number of fibers. Indeed, if the support of $T$ meets the fibers transversally, this would contradict the orthogonality condition.

It follows that $T$ is a fiber sheaf. Suppose that $\mu_{\max }(T) \leq 0$ on the fibers where $T$ is supported. In this case, $T$ would be $\Psi$-WIT 1 and applying $\Psi$ we would find that $\operatorname{Hom}(\Psi(T), E)=\operatorname{Hom}(T, \Phi(E)) \neq 0$, contradicting the torsion freeness of $E$. Indeed $\Psi(T)$ is a fiber sheaf as well. It $\mu_{\max }(T)>0$ for some fiber, then $T$ would admit a sub-line bundle of non positive slope and the same argument apply.

Then $\Phi(E)$ is torsion free and we have to prove that it is a vector bundle. Suppose this is not the case and consider the exact sequence

$$
0 \longrightarrow \Phi(E) \longrightarrow(\Phi(E))^{\vee \vee} \longrightarrow T^{\prime} \longrightarrow 0
$$

where $T^{\prime}$ is zero dimensional. Since $T^{\prime}$ is $\Psi$ - $\mathrm{WIT}_{0}$ and $\Psi\left(T^{\prime}\right)$ is a sheaf concentrated on a finite number of fibers, applying $\Psi$ gives a contradiction to the torsion freeness of $E$.

Proposition 4.5. The indeterminacy locus of $\rho$ is the projective bundle over $X \times M_{\widehat{X}}(\hat{u})$ associated to the universal extension of the form $\operatorname{PExt}^{1}\left(\mathcal{P}_{x}, F\right)$, for $F$ in $M_{\widehat{X}}(\hat{u})$ and $\mathcal{P}_{x}$ in $\widehat{\widehat{X}} \cong X$.

Proof. Let $E$ be in $M_{\widehat{X}}(\hat{v})$. First, $E$ is not in the indeterminacy locus of $\rho$ if and only if $E$ is $\Phi-\mathrm{WIT}_{0}$. Indeed, if $E$ is $\Phi-\mathrm{WIT}_{0}$, then it is $\Phi-\mathrm{IT}_{0}$ and $\Phi(E)$ is then a locally free sheaf on $X$ of rank $r$, fiber degree $-d$ and it is $(r,-d)^{\perp}$. It follows that 
$\Phi(E)$ is in $M_{X}(v)$. Conversely, the fact that $\Phi(E)$ is in $M_{X}(v)$ tells in particular that $\Phi(E)$ is a sheaf.

Recall that by [2, Lemma 6.1$]$, for all sheaves $E$ on $\widehat{X}$, there exists a unique short exact sequence

$$
0 \longrightarrow A \longrightarrow E \longrightarrow B \longrightarrow 0
$$

where $A$ is $\Phi$-WIT $\mathrm{W}_{0}$ and $B$ is $\Phi$-WIT 1 .

Suppose that the rank of $B$ is positive. Then by the stability of $E$, we get that the slope of $B$ has to be positive as well, but in this case $B$ would not be $\Phi-\mathrm{WIT}_{1}$, by $[2$, Lemma 6.2$]$. Then $B$ is a torsion $\Phi-\mathrm{WIT}_{1}$ sheaf, and then it is a fiber sheaf by $[2$, Lemma 6.3$]$. If $\widehat{X}_{0}$ is a fiber in the support of $B$, then restricting the $\Phi$ to $\widehat{X}_{0}$, we have that $\mu_{\max }(B) \leq 0$, since $B$ is $\Phi-\mathrm{WIT}_{1}$. Then $B$ is a pure fiber sheaf of non-positive maximal slope.

Let $\widehat{X}_{0}$ be a fiber in the support of $B$ and $r_{0}$ and $-d_{0}$ be respectively the rank and the degree of $B$ as a sheaf on $\widehat{X}_{0}$. Recall that $E$ has rank $d$ and fiber degree $r$. Then applying point (1) of Proposition 2.17, we get $r_{0} r+d d_{0} \leq t$. Since $r_{0}>0$, we get that $B$ has rank 1 and degree 0 . Moreover, $B$ cannot be supported on any other fiber, by point (2) of Proposition 2.17. This identifies also the Mukai vector of $A$, up to a twist with a line bundle.

Finally, any extension of the form

$$
0 \longrightarrow F \longrightarrow E \longrightarrow \mathcal{P}_{x} \longrightarrow 0
$$

is stable. Indeed, such an $E$ is torsion free and satisfies the orthogonality condition. $\square$

Now let $U \subset M_{X}(v)$ be the subset where $\varepsilon$ is defined and $\widehat{U} \subset M_{\widehat{X}}(\hat{v})$ the subset where $\rho$ is defined. It is now clear that $\varepsilon(U)=\widehat{U}$ and $\rho(\widehat{U})=U$. Moreover, $\varepsilon \circ \rho=\rho \circ \varepsilon=$ Id. Compare Propositions 4.3 and 4.5 by using Serre duality. A dimension calculation shows that the indeterminacy loci have codimension $r-1$. If $t=r=2$ the indeterminacy loci have codimension 1 , which gives an isomorphism. In any other case, since $r>2$, we are dualizing a projective bundle and then we have a nontrivial Mukai flop and this proves Theorem 4.1.

REMARK 4.6. Suppose that $t \geq r+d$. Then the indeterminacy locus of $\varepsilon$ gets bigger, since it contains all those stable vector bundles $E$ which have "very unstable" restriction to some fiber. Anyway, if $r>2$, it is clear by the description of the indeterminacy loci of $\varepsilon$ and $\rho$ that we would never have an isomorphism for $t \geq r$. For $r>2$, we obtained that the condition $t<r$ is optimal.

REMARK 4.7. If $r=2$, consider the case $t=4$. In this case, there are sheaves $E$ in $M_{X}(v)$ such that the cokernel of the map $E \hookrightarrow E^{\vee \vee}$ has length 2. These sheaves belong to the indeterminacy locus and their locus forms a projective bundle $\mathbb{P} \rightarrow X \times M_{\widehat{X}}(\hat{w})$, where $w=(2, \Lambda, c+2)$. If we go through the proof of Theorem 4.1 , we can define a stratum in the indeterminacy locus of $\rho$ which is parameterized by $\mathbb{P}^{\vee}$. This stratum is indeed given by those semistable sheaves admitting $\mathcal{P}_{x_{1}} \oplus \mathcal{P}_{x_{2}}$ as a quotient. This should lead to a non-trivial flop and then to a contradiction to $[4$, III, Conjecture 4.13].

Appendix A. Continued fractions and the Euclidean algorithm. In this Appendix, we give a proof of Lemma 3.14. We start with a version of the Euclidean 
algorithm for integers $(r, d)$ with $0<d<r$. We start by setting $\left(r_{0}, d_{0}\right)=(r, d)$ and proceed inductively with $\left(r_{i+1}, d_{i+1}\right)=\left(d_{i},\left\lceil\frac{r_{i}}{d_{i}}\right\rceil d_{i}-r_{i}\right)$. The algorithm stops when $d_{n+1}=0$, the corresponding $r_{n+1}$ is the greatest common divisor of $r$ and $d$. We assume from now on that $r$ and $d$ are coprime. We denote the integers $\left\lceil\frac{r_{n}}{d_{n}}\right\rceil$ by $a_{n}$ and remark that $a_{i} \geq 2$. We see that we can recover $r$ and $d$ from the numbers $\left\{a_{i}\right\}_{i=0, \ldots, n}$ since

$$
\frac{r}{d}=\left\lceil a_{0}, a_{1}, \ldots, a_{n}\right\rceil=a_{0}-\frac{1}{a_{1}-\frac{1}{a_{2}-\frac{1}{a_{3}-\frac{1}{\cdots-\frac{1}{a_{n}}}}}} .
$$

Since $(r, d)=1$ there are integers $b$ and $c$ such that $r c+d b=1$. The pair $(b, c)$ is uniquely determined when we assume $0 \leq b<r$. Note that our assumption $0<d<r$ implies $0<b<r$. Lemma 3.14 is now proved by point (4) of the following result.

Lemma A.1. Let $\left\{a_{i}\right\}_{i=0, \ldots, n}$ be integers with $a_{i} \geq 2$. Let $\frac{r}{d}=\left\lceil a_{0}, a_{1}, \ldots, a_{n}\right\rceil$ written in reduced form. Let $(b, c)$ be integers with $r c+d b=1$ and $0 \leq b<r$. The following holds:

1. The number $\frac{r}{d}$ satisfies $\frac{r}{d}>1$.

2. The integer $b$ is positive.

3. If we reverse the order in the continued fraction for $\frac{r}{d}$, then we obtain the continued fraction expression of $\frac{r}{b}$. In short: $\frac{r}{b}=\left\lceil a_{n}, a_{n-1}, \ldots, a_{0}\right\rceil$.

4. We have an equality $a_{n}=\left\lceil\frac{r}{b}\right\rceil$.

Proof. (1) Follows by induction since $\left\lceil a_{n}\right\rceil=a_{n} \geq 2$, and $\left\lceil a_{i-1}, a_{i}, \ldots, a_{n}\right\rceil=$ $a_{i-1}-\frac{1}{\left\lceil a_{i}, \ldots, a_{n}\right\rceil}$.

(2) Since $\frac{r}{d}>1$ we have $0<d<r$. Thus there are no solutions $(b, c)$ of the equation $r c+d b=1$ with $b=0$.

(3) We observe that $\left(\begin{array}{l}r \\ d\end{array}\right)$ is the first column of the $\mathrm{SL}_{2}(\mathbb{Z})$-matrix

$$
A=T^{a_{0}} \cdot S \cdot T^{a_{1}} \cdot S \cdot \ldots T^{a_{n}} \cdot S \quad \text { with } \quad T=\left(\begin{array}{ll}
1 & 1 \\
0 & 1
\end{array}\right), \text { and } S=\left(\begin{array}{rr}
0 & -1 \\
1 & 0
\end{array}\right) .
$$

We write $A=\left(\begin{array}{rr}r & -b^{\prime} \\ d & c^{\prime}\end{array}\right)$ and set $R=\left(\begin{array}{rr}-1 & 0 \\ 0 & 1\end{array}\right)$. We compute that $R \cdot A^{t} \cdot R=$ $\left(\begin{array}{rr}r & -d \\ b^{\prime} & c^{\prime}\end{array}\right)$. On the other hand using the above product presentation for $A$ together with the two matrix equations $R=R^{-1}$ and $R \cdot S^{t} \cdot\left(T^{a_{i}}\right)^{t} \cdot R=T^{a_{i}} \cdot S$ we obtain that:

$$
\begin{aligned}
R \cdot A^{t} \cdot R & =R \cdot S^{t} \cdot\left(T^{a_{n}}\right)^{t} \cdot S^{t} \cdot\left(T^{a_{n-1}}\right)^{t} \cdots \cdots S^{t} \cdot\left(T^{a_{0}}\right)^{t} \cdot R \\
& =\left(R \cdot S^{t} \cdot\left(T^{a_{n}}\right)^{t} \cdot R\right)\left(R \cdot S^{t} \cdot\left(T^{a_{n-1}}\right)^{t} \cdot R\right) \cdots \cdots\left(R \cdot S^{t} \cdot\left(T^{a_{0}}\right)^{t} \cdot R\right) \\
& =T^{a_{n}} \cdot S \cdot T^{a_{n-1}} \cdot S \cdot \ldots T^{a_{0}} \cdot S
\end{aligned}
$$

This implies that $\frac{r}{b^{\prime}}=\left\lceil a_{n}, a_{n-1}, \ldots, a_{0}\right\rceil$. We deduce from $a_{i} \geq 2$ and part (1) that $0<b^{\prime}<r$. Since the matrix $A$ is in $\mathrm{SL}_{2}(\mathbb{Z})$ we have $r c^{\prime}+d b^{\prime}=1$. Thus $b=b^{\prime}$.

(4) Is a direct consequence of the expression $\frac{r}{b}=\left\lceil a_{n}, a_{n-1}, \ldots, a_{0}\right\rceil$ derived in (3). 


\section{REFERENCES}

[1] M. АтіYAн, Vector bundles over an elliptic curve, Proc. London Math. Soc., 7 (1957), pp. 414452.

[2] T. Bridgeland, Fourier-Mukai transforms for elliptic surfaces, J. Reine Angew. Math., 498 (1998), pp. 115-133.

[3] R. Friedman, Rank 2 vector bundles over regular elliptic surfaces, Invent. Math., 96 (1989), pp. 283-332.

[4] R. Friedman, Vector bundles and SO(3)-invariants for elliptic surfaces, J. Am. Math. Soc., 8 (1995), pp. 29-139.

[5] S. I. Gelfand and Yu. I. Manin, Methods of homological algebra, Springer, Berlin, 1997.

[6] G. Hein And D. Ploog, Postnikov stability for complexes, Int. J. Math., 23 (2012), 1250048, $20 \mathrm{pp}$.

[7] G. HeIn And D. Ploog, Postnikov-stability versus semistability of sheaves, Asian J. Math., 18 (2014), pp. 247-262

[8] D. Huybrechts And M. Lehn, The geometry of moduli spaces of sheaves, Aspects of Mathematics, E31, Braunschweig 1997.

[9] A. Marian and D. Oprea, Generic strange duality for $K 3$ surfaces, with an Appendix by Kota Yoshioka, Duke Math. J., 162 (2013), pp. 1463-1501.

[10] K. O'Grady, The weight-two Hodge structure of moduli spaces of sheaves on a K3 surface, J. Algebraic Geom., 6 (1997), pp. 599-644.

[11] A. Polishchuk, Abelian varieties, theta functions and the Fourier transform, Cambridge (2003).

[12] M. POPA, Dimension estimates for Hilbert schemes and effective base point freeness on moduli spaces of vector bundles on curves, Duke Math J., 107 (2001), pp. 469-495.

[13] K. Yoshioka, Moduli spaces of stable sheaves on abelian surfaces, Math. Ann., 321 (2001), pp. $817-884$. 